\title{
Mirror Symmetry and Fano Manifolds
}

Tom Coates, Alessio Corti, Sergey Galkin, Vasily Golyshev, Alexander Kasprzyk

2010 Mathematics Subject Classification. Primary 14J33; Secondary 14J45, 14D07 Keywords. Fano manifolds, mirror symmetry, variations of Hodge structure, LandauGinzburg models.

\section{Contents}

1 Introduction

2 Local systems

3 Local systems from Laurent polynomials

4 Local systems from quantum cohomology

5 Extremal local systems and extremal Laurent polynomials 9

6 Examples in low dimensions

7 Minkowski polynomials and Fano 3-folds 12

8 Fano 4-folds? 13

\section{Introduction}

We give a sketch of mirror symmetry for Fano manifolds and we outline a program to classify Fano 4-folds using mirror symmetry. As motivation, we describe how one can recover the classification of Fano 3-folds from the study of their mirrors. A glance at the table of contents will give a good idea of the topics covered. We take a stripped-down view of mirror symmetry that originated in the work of Golyshev Gol07 and that can also be found in Prz07.

\section{Local systems}

A local system of rank $r$ on a (topological) manifold $B$ is a locally constant sheaf $\mathbb{V}$ of $r$-dimensional $\mathbb{Q}$-vector spaces. To give a local system is equivalent to give its monodromy representation $\rho: \pi_{1}(B, x) \rightarrow$ Aut $\mathbb{V}_{x} \cong G L_{r}(\mathbb{Q})$ where $x \in B$. We 
write $r=\mathrm{rk} \mathbb{V}$. The central theme of this note is the detailed comparison of two different ways that local systems arise in mathematics.

All local systems in this note: (a) support - at least conjecturally - an additional structure such as a (polarised) variation of (pure) Hodge structure, or a structure of an $l$-adic sheaf over a base $B$ defined over a number field. 1; and (b) have an integral structure, for instance they are local systems of free $\mathbb{Z}$-modules. In particular we assume throughout that $\mathbb{V}$ is polarised, i.e. that it carries a nondegenerate symmetric or antisymmetric bilinear form $\psi: \mathbb{V} \otimes \mathbb{V} \rightarrow \mathbb{Q}$.

Let $C$ be a compact topological surface, $S \subset C$ a finite set, and $\mathbb{V}$ a local system on $U=C \backslash S$. We denote by $x \in U$ a point and by $j: U=C \backslash S \hookrightarrow C$ the natural (open) inclusion. If $s \in S$ and $\gamma_{s} \in \pi_{1}(U, x)$ is a loop around $s$, then we write $T_{s}=\rho\left(\gamma_{s}\right) \in$ Aut $\mathbb{V}_{x}$ for the monodromy transformation; $T_{s}$ is defined only up to conjugation, but this will be unimportant in what follows.

Definition 2.1. The ramification of $\mathbb{V}$ is:

$$
\operatorname{rf} \mathbb{V}=\sum_{s \in S} \operatorname{dim}\left(\mathbb{V}_{x} / \mathbb{V}_{x}^{T_{s}}\right) .
$$

If $\mathbb{V}$ as above is a local system on $U=C \backslash S$, and the genus of $C$ is $g$, then, by Euler-Poincaré, $\operatorname{rf} \mathbb{V}+(2 g-2) \mathrm{rk} \mathbb{V}=-\chi\left(C, j_{\star} \mathbb{V}\right)$. If $\mathbb{V}$ is nontrivial irreducible, then $H^{0}\left(C, j_{\star} \mathbb{V}\right)=\mathbb{V}_{x}^{\pi_{1}(U, x)}=(0)$ and, dually, also $H^{2}\left(C, j_{\star} \mathbb{V}\right)=(0)$. Thus, if $C=\mathbb{P}^{1}$ and $\mathbb{V}$ is nontrivial irreducible, then:

$$
\operatorname{rf} \mathbb{V}-2 \operatorname{rk} \mathbb{V}=-\chi\left(\mathbb{P}^{1} ; j_{\star} \mathbb{V}\right)=h^{1}\left(\mathbb{P}^{1} ; j_{\star} \mathbb{V}\right) \geq 0 .
$$

We call the quantity $\mathrm{rf} \mathbb{V}-2 \mathrm{rk} \mathbb{V}$ the ramification index of $\mathbb{V}$. Even from a purely topological perspective, local systems with ramification index zero seem special. As far as we know, to date there has been no systematic study of $l$-adic sheaves on $\mathbb{P}^{1}$ of ramification zero.

\section{Local systems from Laurent polynomials}

Local systems arise classically in algebraic geometry as the cohomology groups of the fibers of a morphism $f: X \rightarrow B$.

The classical period of a Laurent polynomial. We discuss the special case where $f:\left(\mathbb{C}^{\times}\right)^{n} \rightarrow \mathbb{C}$ is a Laurent polynomial in $n$ variables, that is, an element of the polynomial ring $\mathbb{C}\left[x_{1}, x_{1}^{-1}, \ldots, x_{n}, x_{n}^{-1}\right]$ where $x_{1}, \ldots, x_{n}$ are the standard co-ordinates on $\left(\mathbb{C}^{\times}\right)^{n}$.

\footnotetext{
${ }^{1}$ It is natural to imagine that local systems with additional structures (realisations) subject to natural comparisons would be the object of a category of "quantum" motivic sheaves with a construction akin to Del89, Jan90, see [KKP08]. It is interesting to wonder what a Grothendieckstyle definition of such a category might look like, and what it might mean.
} 
Definition 3.1. Let $f:\left(\mathbb{C}^{\times}\right)^{n} \rightarrow \mathbb{C}$ be a Laurent polynomial. The classical period of $f$ is:

$$
\pi_{f}(t)=\left(\frac{1}{2 \pi \mathrm{i}}\right)^{n} \int_{\left|x_{1}\right|=\cdots=\left|x_{n}\right|=1} \frac{1}{1-t f\left(x_{1}, \ldots, x_{n}\right)} \frac{d x_{1}}{x_{1}} \cdots \frac{d x_{n}}{x_{n}}
$$

Theorem 3.2. The classical period satisfies an ordinary differential equation $L$. $\pi_{f}(t) \equiv 0$, where $L \in \mathbb{C}\langle t, D\rangle$ is a polynomial differential operator and $D=t \frac{d}{d t}$.

Proof. In short: our period $\pi_{f}(t)$ is a specialisation of integrals which are solutions of the differential systems introduced in GZK89, for which we recommend the survey Sti07]. We next explain this in greater detail. Let $P \subset \mathbb{Z}^{n}$ be the Newton polytope of $f$ and denote by $m_{0}, \ldots, m_{N} \in P \cap \mathbb{Z}^{n}$ the lattice points in $P$. If $P$ does not contain the origin then the classical period is constant and there is nothing to prove, so we assume that $m_{0}=0$. Write:

$$
f=\sum_{i=0}^{N} a_{i} x^{m_{i}}
$$

Reparametrizing $t$ if necessary, we reduce to the case where $a_{0}=0$. Denote by $\iota: \mathbb{Z}^{n} \hookrightarrow \mathbb{Z}^{n+1}$ the affine embedding "at height 1 ": $\iota(m)=(1, m)$. Write $\mathbf{m}_{i}=\iota\left(m_{i}\right), 0 \leq i \leq N$, and let $A: \mathbb{Z}^{N+1} \rightarrow \mathbb{Z}^{n+1}$ be the homomorphism that maps the standard basis vector $\mathbf{e}_{i}$ to $\mathbf{m}_{i}, 0 \leq i \leq N$. If:

$$
g=\sum_{i=0}^{N} u_{i} x^{m_{i}}
$$

is the generic Laurent polynomial with Newton polytope $P$, then it is well-known Bat94, Sti07 that the period:

$$
\Phi_{g}\left(u_{0}, \ldots, u_{N}\right)=\left(\frac{1}{2 \pi \mathrm{i}}\right)^{n} \int \frac{1}{g} \frac{d x_{1}}{x_{1}} \cdots \frac{d x_{n}}{x_{n}}
$$

satisfies the holonomic differential system $2 \operatorname{gkz}(A, \mathbf{c})$ where $\mathbf{c}=(-1,0, \ldots, 0)$ Sti07, §2.5]. To get the operator $L$, restrict the coefficients to $u_{i}=a_{i}$ for $i>0$, change the variable $u_{0}$ to $t=-1 / u_{0}$, and note that $\pi_{f}(t)=u_{0} \Phi_{g}\left(u_{0}, a_{1}, \ldots, a_{n}\right)$.

${ }^{2}$ That is, the system of differential equations:

$$
\left\{\begin{array}{l}
0=\prod_{l_{i}<0}\left(\frac{\partial}{\partial u_{i}}\right)^{-l_{i}}-\prod_{l_{i}>0}\left(\frac{\partial}{\partial u_{i}}\right)^{l_{i}} \\
0=-\mathbf{c}+\mathbf{m}_{0} u_{0} \frac{\partial}{\partial u_{0}}+\cdots+\mathbf{m}_{N} u_{N} \frac{\partial}{\partial u_{N}}
\end{array} \quad \text { for } \mathbf{l}=\left(l_{1}, \ldots, l_{N+1}\right) \in \operatorname{Ker} A\right.
$$

More precisely the period satisfies the extended GKZ system of HKTY95 §3.3] or, equivalently, the better behaved $\mathrm{GKZ}$ system of $[\mathrm{BH}]$. In the important case when $P$ is a reflexive polytope, the standard GKZ is the same as the better behaved GKZ. The rank of the local system of solutions of the better behaved system is always the normalised volume $\operatorname{Vol} P$. 
Definition 3.3. The Picard-Fuchs operator $L_{f} \in \mathbb{C}\langle t, D\rangle$ is the operator:

$$
L_{f}=\sum_{j=0}^{k} p_{j}(t) D^{j} \quad p_{j} \in \mathbb{C}[t]
$$

such that $L_{f} \cdot \pi_{f} \equiv 0$, where $k$ is taken to be as small as possible and, once $k$ is fixed, we choose $L_{f}$ so that $\operatorname{deg} p_{k}$ is as small as possible. This defines $L_{f}$ uniquely up to multiplication by a constant. We say that the order ord $L_{f}$ of $L_{f}$ is $k$, and the degree $\operatorname{deg} L_{f}$ is the maximum of $\operatorname{deg} p_{0}, \operatorname{deg} p_{1}, \ldots, \operatorname{deg} p_{k}$.

It is clear from what we said above that ord $L_{f} \leq \operatorname{Vol} P$.

Remark 3.4. The local system Sol $L_{f}$ is an irreducible summand of the polarised variation of Hodge structure $\operatorname{gr}_{n-1}^{W} R^{n-1} f_{!} \mathbb{Z}_{\left.\mathbb{C}^{\times}\right)^{n}}$. By [Del71, Thm 4.5], $L_{f}$ has regular singularities.

How to compute the Picard-Fuchs operator and the ramification. Consider the period sequence $\left(c_{m}\right)_{m \geq 0}$, where $c_{m}=\operatorname{coeff}_{\mathbf{1}}\left(f^{m}\right)$. Expanding $\pi_{f}(t)$ as a power series in $t$ and applying the residue theorem $n$ times yields:

$$
\pi_{f}(t)=\sum_{m=0}^{\infty} c_{m} t^{m}
$$

Consider a polynomial differential operator $L=\sum t^{k} P_{k}(D)$ where $P_{k}(D) \in \mathbb{C}[D]$ is a polynomial in $D$; then $L \cdot \pi_{f} \equiv 0$ is equivalent to the linear recursion relation $\sum P_{k}(m-k) c_{m-k}=0$. In practice, to compute $L_{f}$ one uses knowledge of the first few terms of the period sequence and linear algebra to guess the recursion relation; note that the computation of $c_{m}$, say for $1 \leq m \leq 600$, is very expensive. Given $L_{f}$, one can compute $\operatorname{rf}\left(\mathrm{Sol} L_{f}\right)$ algorithmically using elementary Fuchsian theory.

Example 3.5. If $f(x, y)=x+y+x^{-1} y^{-1}$, then:

$$
\pi_{f}(t)=\sum_{m \geq 0} \frac{(3 m) !}{(m !)^{3}} t^{3 m}
$$

The coefficients satisfy the recursion relation:

$$
m^{2} c_{3 m}-3(3 m-1)(3 m-2) c_{3 m-3}=0
$$

and, by what we said, this is equivalent to:

$$
\left[D^{2}-27 t^{3}(D+1)(D+2)\right] \pi_{f}=0
$$

Studying this ODE, one finds that the ramification defect $\operatorname{rf}\left(\operatorname{Sol} L_{f}\right)-2 \operatorname{rk}\left(\operatorname{Sol} L_{f}\right)$ is zero.

Example 3.6. Consider $f(x, y)=x+x y+y+x^{-1} y^{-1}$. In this case:

$$
\begin{aligned}
L_{f}=8 D^{2}-t D-t^{2}(5 D+8) & (11 D+8)-12 t^{3}\left(30 D^{2}+78 D+47\right) \\
- & 4 t^{4}(D+1)(103 D+147)-99 t^{5}(D+1)(D+2)
\end{aligned}
$$

and the ramification defect $\operatorname{rf}\left(\operatorname{Sol} L_{f}\right)-2 \operatorname{rk}\left(\operatorname{Sol} L_{f}\right)=1$. 


\section{Local systems from quantum cohomology}

Local systems also arise in the study of quantum cohomology, as solutions of the regularised quantum differential equation. When $X$ is a Fano manifold, the space of solutions of the regularised quantum differential equation for $X$ defines a local system on $\mathbb{P}^{1} \backslash S$.

Fano manifolds. Recall that a complex projective manifold $X$ of complex dimension $n$ is called Fano if the anticanonical line bundle $-K_{X}=\wedge^{n} T_{X}$ is ample. If $n=2, X$ is called a del Pezzo surface. It is well-known that a del Pezzo surface is isomorphic to $\mathbb{P}^{1} \times \mathbb{P}^{1}$ or the blow up of $\mathbb{P}^{2}$ in $\leq 8$ general points: thus, there are 10 deformation families of Fano manifolds in two dimensions. There are 105 deformation families of 3-dimensional Fano manifolds: 17 families with $b_{2}=1$ and 88 families with $b_{2} \geq 2$ [sk77, Isk78, Tak89, MM04]. We state a theorem of Mori that plays a crucial role in what follows:

Theorem 4.1. Let $X$ be a Fano manifold. Denote by $\mathrm{NE} X \subset H_{2}(X ; \mathbb{R})$ the Mori cone of $X$ : that is, the convex cone generated by (classes of) algebraic curves $C \subset X$. Then NE $X$ is a rational polyhedral cone.

The quantum period of a Fano manifold. When $X$ is Fano, denote by $X_{0, k, m}$ the moduli space of stable morphisms $f:\left(C, x_{1}, \ldots, x_{k}\right) \rightarrow X$ where $C$ is a curve of genus 0 with $k$ marked points $x_{1}, \ldots, x_{k} \in C$, and $\operatorname{deg} f^{\star}\left(-K_{X}\right)=m$. This moduli space has virtual dimension $m-3+n+k$. Here we are mainly interested in $X_{0,1, m}$ and the evaluation morphism at the marked point:

$$
\mathrm{ev}: X_{0,1, m} \rightarrow X
$$

Denote by $\psi$ the first Chern class of the universal cotangent line bundle on $X_{0,1, m}$, that is, the relative dualising sheaf $\omega_{\pi}$ of the forgetful morphism $\pi: X_{0,1, m} \rightarrow$ $X_{0,0, m}$.

Definition 4.2. The quantum period of $X$ is the power series $G_{X}(t)=\sum_{m>0} p_{m} t^{m}$ where $p_{0}=1, p_{1}=0$, and $p_{m}=\int_{X_{0,1, m}} \psi^{m-2} \mathrm{ev}^{\star}(\mathrm{pt})$ for $m \geq 2$. The sequence $\left(p_{m}\right)_{m \geq 0}$ is the quantum period sequence.

Theorem 4.3. The quantum period of a Fano manifold $X$ satisfies a ordinary differential equation $Q \cdot G_{X}(t) \equiv 0$, where $Q \in \mathbb{Z}\langle t, D\rangle$ is a polynomial differential operator and $D=t \frac{d}{d t}$.

Proof. In short: our quantum period $G_{X}(t)$ is a specialisation of one component of the small $J$-function. The result then follows from general properties of quantum cohomology going back to Dijkgraaf. We next recall the relevant facts from the theory of quantum cohomology $\sqrt{3}$ and explain this in greater detail.

In what follows we denote by $X_{0, k, \beta}$ the moduli space of stable morphisms of class $\beta \in \mathrm{NE} X \cap H_{2}(X, \mathbb{Z})$. Recall that the small quantum product $a * b$ of even

\footnotetext{
${ }^{3}$ See Gue08, Man99] for more comprehensive treatments.
} 
degree cohomology classes $a, b \in H^{\bullet}(X ; \mathbb{C})$ is defined by the following formula, which is to hold for all $c \in H^{\bullet}(X ; \mathbb{C})$ :

$$
(a * b, c)=\sum_{\beta \in \mathrm{NE} X \cap H_{2}(X ; \mathbb{Z})} q^{\beta}\langle a, b, c\rangle_{0,3, \beta}
$$

where $(a, b)=\int_{X} a \cup b$ is the Poincaré pairing, $q^{\beta}$ lies in the group ring $\mathbb{C}\left[H_{2}(X ; \mathbb{Z})^{4}\right.$, and:

$$
\langle a, b, c\rangle_{0,3, \beta}=\int_{X_{0,3, \beta}} \operatorname{ev}_{1}^{\star}(a) \cup \operatorname{ev}_{2}^{\star}(b) \cup \operatorname{ev}_{3}^{\star}(c)
$$

is the 3-point correlator. The structure of the small quantum product is equivalent to an integrable algebraic connection $\nabla$ on:

- the trivial bundle with fiber the even part $H^{\mathrm{ev}}(X ; \mathbb{C})$ of $H^{\bullet}(X ; \mathbb{C})$, over

- the torus $\mathbb{T}=\operatorname{Spec} \mathbb{C}\left[H_{2}(X, \mathbb{Z})\right]$.

In other words $\mathbb{T}$ is the torus with character group $\operatorname{Hom}_{\text {groups }}\left(\mathbb{T}, \mathbb{C}^{\times}\right)=H_{2}(X ; \mathbb{Z})$, co-character group Hom groups $\left(\mathbb{C}^{\times}, \mathbb{T}\right)=H^{2}(X ; \mathbb{Z})$, and group of $\mathbb{C}$-valued points $\mathbb{T}(\mathbb{C})=\mathbb{C}^{\times} \otimes H^{2}(X ; \mathbb{Z})$. Note that Lie $\mathbb{T}=H^{2}(X ; \mathbb{C})$. The connection $\nabla$ is defined by:

$$
\nabla_{X} s=X \cdot s-X * s \quad \text { where } s: \mathbb{T} \rightarrow H^{\mathrm{ev}}(X ; \mathbb{C}) \text { and } X \in \operatorname{Lie} \mathbb{T}=H^{2}(X ; \mathbb{C}) .
$$

The fact that this connection is algebraic globally on $\mathbb{T}$ (in fact, the coefficients of the connection are polynomials) follows from the fact that quantum cohomology is graded and that $-K_{X}>0$ on $\mathrm{NE} X$. The fact that the connection is integrable (flat) is a fundamental property of quantum cohomology: it follows from the WDVV equations. Integrability means that the action of Lie $\mathbb{T}$ on $M=\left\{s: \mathbb{T} \rightarrow H^{\mathrm{ev}}(X ; \mathbb{C})\right\}$ extends to an action of the ring $D$ of differential operators on $\mathbb{T}$ : in other words $M$ is a $D$-module, called the quantum $D$-module. $M$ therefore defines a $\mathcal{D}$-module $\mathcal{M}$, that is, a sheaf of modules $\mathcal{M}$ over the sheaf of differential operators $\mathcal{D}$ on $\mathbb{T}$. In general, given a $\mathcal{D}$-module $\mathcal{M}$, one can form the local system $\underline{\operatorname{Hom}}_{\mathcal{D}}(\mathcal{M}, \mathcal{O})$ of solution 5 of $\mathcal{M}$. Sections of this local system tautologically satisfy algebraic PDEs.

Recall that the small $J$-function of $X$ is:

$$
J_{X}(q)=1+\sum_{\beta \in \mathrm{NE}} \sum_{\substack{X \cap H_{2}(X ; \mathbb{Z}) \\ \beta \neq 0}} q^{\beta} J_{\beta} \in H^{\mathrm{ev}}(X ; \mathbb{C})
$$

where $J_{\beta}=\mathrm{ev}_{\star}\left(\frac{1}{1-\psi}\right)$, ev: $X_{0,1, \beta} \rightarrow X$ is the evaluation map at the marked point, and we expand $\frac{1}{1-\psi}$ as a power series in $\psi$. It is well-known that $J_{X}(q)$ is a solution of the quantum $\mathcal{D}$-module and therefore it tautologically satisfies an

\footnotetext{
${ }^{4}$ In general we should work with the subgroup $H_{2}(X)^{\text {alg }} \subset H_{2}(X)$; here and in the rest of the paragraph we use the fact that if $X$ is Fano manifold then $H_{2}(X)=H_{2}^{\text {alg }}(X)$.

${ }^{5}$ Here $\mathcal{O}$ and $\mathcal{M}$ are sheaves of $\mathcal{D}$-modules in the analytic topology on $\mathbb{T}$, and $\underline{H o m}$ is the sheaf of homomorphisms.
} 
algebraic PDE. Note that $J_{X}(q)$ is cohomology-valued but it makes sense to take its degree-zero component $J_{X}^{0}(q) \in H^{0}(X ; \mathbb{C})$; we can regard $J_{X}^{0}(q)$ as a $\mathbb{C}$-valued function, because $H^{0}(X ; \mathbb{C})$ is canonically generated by the identity class 1 .

Finally, the anticanonical class $-K_{X} \in H^{2}(X ; \mathbb{Z})$ is a co-character of $\mathbb{T}$, that is, $-K_{X}$ gives a group homomorphism which we denote $\kappa: \mathbb{C}^{\times} \rightarrow \mathbb{T}$. Since $G_{X}(t)=$ $J_{X}^{0} \circ \kappa(t)$, where $t$ is the co-ordinate function on $\mathbb{C}^{\times}$, the discussion above makes it clear that $G_{X}(t)$ satisfies an algebraic ODE.

Definition 4.4. The quantum differential operator of $X$ is the operator $Q_{X} \in$ $\mathbb{Z}\langle t, D\rangle$ of lowest order, as in Definition 3.3. such that $Q_{X} \cdot G_{X}(t) \equiv 0$.

How to compute $\boldsymbol{Q}_{\boldsymbol{X}}$. In practice one starts by fixing a basis $\left\{T^{a}\right\}$ of $H^{\mathrm{ev}}(X ; \mathbb{Z})$ with $T^{0}=1$ the identity class. Let $M=M(t)$ be the matrix of quantum multiplication by $-K_{X}$ in this basis, written as a function on $\mathbb{C}^{\times}$by composing with $\kappa: \mathbb{C}^{\times} \rightarrow \mathbb{T}$. Next consider the differential equation on $\mathbb{C}^{\times}:$

$$
\left\{\begin{array}{l}
D \Psi(t)=\Psi(t) M(t) \\
\Psi(0)=\mathrm{I}
\end{array}\right.
$$

for $\Psi: \mathbb{C}^{\times} \rightarrow \operatorname{End}\left(H^{\mathrm{ev}}(X ; \mathbb{C})\right)$ a matrix. (Note: tautologically, the differential $\kappa_{\star}:$ Lie $\mathbb{C}^{\times} \rightarrow$ Lie $\mathbb{T}$ sends $D=t \frac{d}{d t}$ to $-K_{X} \in H^{2}(X ; \mathbb{C})=$ Lie $\mathbb{T}$. $)$ Then the first column of $\Psi$ is $J_{X} \circ \kappa(t)$; the first entry of the first column is our quantum period $G_{X}(t)$.

Example 4.5. Consider $X=\mathbb{P}^{2}$ with cohomology ring $\mathbb{C}[P] / P^{3}$, where $P$ is the first Chern class of $\mathcal{O}(1)$. Choose the basis $1,-K_{X}=3 P, K_{X}^{2}=9$ pt for the cohomology. The matrix of quantum multiplication by $-K_{X}$, in this basis, is:

$$
M=\left(\begin{array}{ccc}
0 & 0 & 27 t^{3} \\
1 & 0 & 0 \\
0 & 1 & 0
\end{array}\right)
$$

where the coefficient of $t^{3}$ in the upper right corner of the matrix is calculated as a nontrivial Gromov-Witten number:

$$
\left\langle-K_{X} *\left(K_{X}^{2}\right), \mathrm{pt}\right\rangle_{0,3,[\text { line }]}=3\left\langle K_{X}^{2}, \mathrm{pt}\right\rangle_{0,2,[\text { line }]}=27\langle\mathrm{pt}, \mathrm{pt}\rangle_{0,2,[\text { line }]}=27
$$

Next we consider the system:

$$
D\left(s_{0}, s_{1}, s_{2}\right)=\left(s_{0}, s_{1}, s_{2}\right) M
$$

The column $s_{0}$ is annihilated by the differential operator $Q_{X}=D^{3}-27 t^{3}$, and so $G_{X}(t)=\sum_{m=0}^{\infty} \frac{t^{3 m}}{(m !)^{3}}$. 
Computing $G_{X}$ using the quantum Lefschetz theorem. We explain how to calculate the quantum period of a Fano complete intersection in a toric manifold using the quantum Lefschetz theorem of Kim, Lee, and Coates-Givental. For us, a toric variety is a GIT quotient $X=\mathbb{C}^{r} / / \chi\left(\mathbb{C}^{\times}\right)^{b}$ where $\left(\mathbb{C}^{\times}\right)^{b}$ acts via the composition of a group homomorphism $\rho:\left(\mathbb{C}^{\times}\right)^{b} \rightarrow\left(\mathbb{C}^{\times}\right)^{r}$ with the canonical action of $\left(\mathbb{C}^{\times}\right)^{r}$ on $\mathbb{C}^{r}$. The group homomorphism $\rho$ is given dually by a $b \times r$ integral matrix:

$$
D=\left(D_{1}, \ldots D_{r}\right): \mathbb{Z}^{r} \rightarrow \mathbb{Z}^{b}
$$

that we call the weight data of the toric variety $X$. The weight data alone do not determine $X$ : it is necessary to choose a stability condition, i.e. a $\left(\mathbb{C}^{\times}\right)^{b}$-linearized line bundle $L$ on $\mathbb{C}^{r}$. This choice is equivalent to the choice of a character $\chi \in \mathbb{Z}^{b}$ of $\left(\mathbb{C}^{\times}\right)^{b}$; denoting by $L_{\chi}$ the corresponding line bundle, we have:

$$
H^{0}\left(\mathbb{C}^{r} ; L_{\chi}\right)^{\left(\mathbb{C}^{\times}\right)^{b}}=\left\{f \in \mathbb{C}\left[x_{1}, \ldots, x_{r}\right]: f(\lambda x)=\chi(\lambda) f(x) \text { for all } \lambda \in\left(\mathbb{C}^{\times}\right)^{b}\right\}
$$

Having made this choice, the set of stable points is:

$$
U^{s}(\chi)=\left\{\mathbf{a} \in \mathbb{C}^{r}: \exists N \gg 0 \text { and } \exists f \in H^{0}\left(\mathbb{C}^{r} ; L_{\chi}^{\otimes N}\right)^{\left(\mathbb{C}^{\times}\right)^{b}} \text { such that } f(\mathbf{a}) \neq 0\right\}
$$

The set of $\chi \in \mathbb{Z}^{b}$ for which $U^{s}(\chi)$ is non-empty generates a rational polyhedral cone in $\mathbb{R}^{b}$ equipped with a partition into locally closed rational polyhedral chambers defined by requiring that $U^{s}(\chi)$ depends only on the chamber containing $\chi$. We always choose $\chi$ in the interior of a chamber of maximal dimension, and then define $X=U^{s}(\chi) /\left(\mathbb{C}^{\times}\right)^{b}$. Under the identification $\mathbb{Z}^{b}=H^{2}(X ; \mathbb{Z})=\operatorname{Pic}(X)$ the chamber containing $\chi$ is identified with the ample cone $\operatorname{Amp} X$; in this way too we regard the columns $D_{i}$ of the weight data $D$ as elements of $H^{2}(X)$. The appropriate Euler sequence shows that $-K_{X}=\sum_{i=1}^{r} D_{i}$.

Theorem 4.6. Giv98] Let $X$ be a toric Fano manifold. Then

$$
G_{X}(t)=\sum_{\mathbf{k} \in \mathbb{Z}^{b} \cap \mathrm{NE} X} t^{-K_{X} \cdot \mathbf{k}} \frac{1}{\left(D_{1} \cdot \mathbf{k}\right) ! \cdots\left(D_{r} \cdot \mathbf{k}\right) !}
$$

Theorem 4.7. Let $\mathbb{F}$ be a Fano toric manifold and let $L_{1}, \ldots, L_{c}$ be nef line bundles on $\mathbb{F}$ such that $A=-\left(K_{\mathbb{F}}+\sum_{i=1}^{c} L_{i}\right) \in \operatorname{Amp} \mathbb{F}$. Let $X$ be a smooth complete intersection of codimension $c$ in $X$, defined by the equation $f_{1}=\cdots=f_{c}=0$ where $f_{i} \in H^{0}\left(\mathbb{F} ; L_{i}\right)$. Let:

$$
F_{X}(t)=\sum_{\mathbf{k} \in \mathbb{Z}^{b} \cap \mathrm{NEF}} t^{A \cdot \mathbf{k}} \frac{\left(L_{1} \cdot \mathbf{k}\right) ! \cdots\left(L_{c} \cdot \mathbf{k}\right) !}{\left(D_{1} \cdot \mathbf{k}\right) ! \cdots\left(D_{r} \cdot \mathbf{k}\right) !}
$$

and let $a_{1}$ be such that $F_{X}=1+a_{1} t+O\left(t^{2}\right)$. Then $G_{X}(t)=\exp \left(-a_{1} t\right) F_{X}(t)$.

Proof. Combine Theorem 4.6 with [CG07]. 
The regularised quantum period and mirror symmetry. The operator $Q_{X}$ has a pole of order 2 (an irregular singularity) at $\infty$, thus it cannot directly be compared with $L_{f}$. This suggests the following definitions:

Definition 4.8. The regularised quantum period is the Fourier-Laplace transform $\widehat{G}_{X}(t)=\sum(m !) p_{m} t^{m}$ of the quantum period $G_{X}(t)$. The regularised quantum differential operator of $X$ is the operator $\widehat{Q}_{X} \in \mathbb{Z}\langle t, D\rangle$ of lowest order, as in Definition 3.3. such that $\widehat{Q}_{X} \cdot \widehat{G}_{X}(t) \equiv 0$.

Definition 4.9. The Laurent polynomial $f$ is mirror-dual to the Fano manifold $X$ if $\pi_{f}(t)=\widehat{G}_{X}(t)$ or, equivalently, if $L_{f}=\widehat{Q}_{X}$.

With this definition a Fano manifold has infinitely many mirrors if it has any at all. The relationship between different mirrors of del Pezzo surfaces is investigated in GU10, CG12, where it is shown that the different mirror Laurent polynomials $f$ are related by cluster transformations, and together define a global function on a cluster variety.

\section{Extremal local systems and extremal Laurent polynomials}

Which local systems arise from the quantum cohomology of Fano manifolds? Golyshev first made the observation that there are effective bounds on the ramification of the regularised quantum local system $\mathbb{V}=\operatorname{Sol} \widehat{Q}_{X}$ of a Fano manifold $X$.

Definition 5.1. Gol A local system $\mathbb{V}$ on $C=\mathbb{P}^{1} \backslash S$ is extremal if it is irreducible, nontrivial, and $\operatorname{rf} \mathbb{V}=2 \mathrm{rk} \mathbb{V}$. A Laurent polynomial $f$ is extremal if the local system Sol $L_{f}$ of solutions of the ODE $L_{f} \cdot() \equiv 0$ is extremal. We write ELP for "extremal Laurent polynomial".

The regularised quantum local system of any 3-dimensional Fano manifold is extremal. We believe that extremal motivic sheaves and Laurent polynomials are interesting in their own right. It would be nice to work out a topological classification of integral polarised extremal local systems.

Example 5.2. Consider a semistable rational elliptic surface $f: X \rightarrow \mathbb{C}$. In general $f$ has 12 singular fibers. Beauville Bea82 classified surfaces with the smallest possible number, 4 , of singular fibers. On each of these $X$, it is easy to find an open set $\left(\mathbb{C}^{\times}\right)^{2} \cong U \subset X$ such that $\left.f\right|_{U}$ is an extremal Laurent polynomial.

\section{Examples in low dimensions}

We describe two classes of Laurent polynomials: Minkowski polynomials (MPs) and Hodge-Tate polynomials. (For simplicity we describe these only when the number of variables involved is 2 or 3.) MPs are especially nice because: (a) they are, experimentally and conjecturally, of low ramification; and (b) any 3dimensional Fano manifold with very ample tangent bundle is mirror-dual to a MP. 
The Minkowski ansatz. Let $P$ be a lattice polytope. Then $P \cap \mathbb{Z}^{n}$ generates an affine lattice whose underlying lattice we denote by Lattice $(P)$.

Definition 6.1. A lattice polytope $P$ is admissible if the relative interior of $P$ contains no lattice points. A lattice polytope $P \subset \mathbb{R}^{n}$ is reflexive if the following two conditions hold:

(a) Int $P \cap \mathbb{Z}^{n}=\{\mathbf{0}\}$;

(b) the polar polytope:

$$
P^{*}=\left\{f \in\left(\mathbb{R}^{n}\right)^{*}:\langle f, v\rangle \geq-1 \text { for all } v \in P\right\}
$$

is a lattice polytope.

Definition 6.2. Let $Q \subset \mathbb{R}^{n}$ be a lattice polytope. A lattice Minkowski decomposition of $Q$ is a decomposition of $Q$ as a Minkowski sum $Q=R+S$ of lattice polytopes $R, S$ such that Lattice $(Q)=\operatorname{Lattice}(R)+\operatorname{Lattice}(S)$.

Fix a reflexive polytope $P \subset \mathbb{R}^{n}$ of dimension $\leq 3$. We describe a recipe, the Minkowski ansatz, to write down Laurent polynomials:

$$
f=\sum_{\mathbf{m} \in P \cap \mathbb{Z}^{n}} a_{\mathbf{m}} x^{\mathbf{m}}
$$

with Newt $(f)=P$. We need to explain how to choose the coefficients $a_{\mathbf{m}}$. In all cases we take $a_{\mathbf{0}}=0$; this is a normalisation choice that corresponds to the fact that $p_{1}=0$. If $F \subset P$ is a face of $P$, the face term corresponding to $F$ is the Laurent polynomial:

$$
f_{F}=\sum_{\mathbf{m} \in F \cap \mathbb{Z}^{n}} a_{\mathbf{m}} x^{\mathbf{m}}
$$

If $P$ is a reflexive polygon then we just need to specify the edge terms. If $E=$ $[\mu, \mu+e \nu]$ is an edge of $P$, where $\nu$ is primitive, we take the corresponding term to be $f_{E}=x^{\mu}\left(1+x^{\nu}\right)^{e}$. If $P$ is a reflexive 3 -tope, then we treat the edges as just said. It remains to specify the face terms $f_{F}$. First, lattice Minkowski decompose each face into irreducibles:

$$
F=F_{1}+\cdots+F_{r}
$$

We say that such a decomposition is admissible if all $F_{i}$ are admissible. Assuming that each face of $P$ has an admissible decomposition, fix such a decomposition: then we take the face term to be: $f_{F}=\prod f_{F_{i}}$ where $f_{F_{i}}$ is given by putting coefficients on the edges of $F_{i}$ exactly as above. Note that the Minkowksi ansatz can associate to a reflexive 3-tope $P$ more than one Laurent polynomial (if one or more faces of $P$ admit more than one admissible decomposition), or exactly one Laurent polynomial (if every face of $P$ admits a unique admissible decomposition), or no Laurent polynomial (if some face of $P$ admits no admissible decomposition). 
MP in 2 variables. There are 16 reflexive polygons and each supports exactly one MP. This gives 16 MPs but only 8 distinct (classical) period sequences. These are the quantum period sequences of the del Pezzo surfaces of degree $\geq 3$, that is, of the del Pezzo surfaces with very ample anti-canonical bundle. The 8 period sequences are extremal with two exceptions: the first we already met in Example 3.6 (the mirror of $\mathbb{F}_{1}$ ), and the other is:

Example 6.3 (the mirror of $\mathrm{dP}_{7}$ ). $f(x, y)=x+y+x^{-1}+y^{-1}+x^{-1} y^{-1}$. Here:

$$
\begin{array}{r}
L_{f}=7 D^{2}+t D(31 D-3)- \\
-t^{2}\left(85 D^{2}+238 D+112\right)-2 t^{3}\left(358 D^{2}+785 D+425\right) \\
-2 t^{4}(D+1)(669 D+970)-731 t^{5}(D+1)(D+2)
\end{array}
$$

and the ramification defect $\operatorname{rf}\left(\operatorname{Sol} L_{f}\right)-2 \operatorname{rk}\left(\operatorname{Sol} L_{f}\right)$ is equal to 1 .

MP in 3 variables. In 3 variables, we have (http://www.fanosearch.net):

- there are 4,319 reflexive 3-topes KS98;

- they have 344 distinct facets, and these have 79 lattice Minkowski irreducible pieces;

- of these, the admissible ones are $A_{n}$-triangles for $1 \leq n \leq 8$;

- MPs supported on reflexive 3-topes give rise to only 165 (classical) period sequences. They are all extremal.

Example 6.4. Consider the reflexive polytope in $\mathbb{R}^{3}$ with vertices given by the columns of:

$$
\left(\begin{array}{rrrrrr}
1 & 0 & 0 & -2 & -3 & -1 \\
0 & 1 & 0 & 0 & -1 & -1 \\
0 & 0 & 1 & -1 & -1 & 1
\end{array}\right)
$$

(This is the polytope with id 519664 in the GRDB database of toric canonical Fano 3-folds [BK].) The pentagonal facet has two Minkowski decompositions, and hence the polytope supports two Minkowski polynomials:

$$
\begin{aligned}
& f_{1}=x+y+z+3 x^{-1}+x^{-1} y^{-1} z+x^{-2} z^{-1}+2 x^{-2} y^{-1}+x^{-3} y^{-1} z^{-1} \\
& f_{2}=x+y+z+2 x^{-1}+x^{-1} y^{-1} z+x^{-2} z^{-1}+2 x^{-2} y^{-1}+x^{-3} y^{-1} z^{-1}
\end{aligned}
$$

The classical periods associated to $f_{1}$ and $f_{2}$ begin as:

$$
\begin{aligned}
& \pi_{1}(t)=1+6 t^{2}+90 t^{4}+1860 t^{6}+44730 t^{8}+1172556 t^{10}+\cdots \\
& \pi_{2}(t)=1+4 t^{2}+60 t^{4}+1120 t^{6}+24220 t^{8}+567504 t^{10}+\cdots
\end{aligned}
$$

and the corresponding Picard-Fuchs operators are:

$$
\begin{aligned}
& L_{1}=144 t^{4} D^{3}+864 t^{4} D^{2}+1584 t^{4} D-40 t^{2} D^{3}+864 t^{4} \\
& -120 t^{2} D^{2}-128 t^{2} D+D^{3}-48 t^{2} \\
& \begin{aligned}
L_{2}=128 t^{4} D^{3}+768 t^{4} D^{2}+1408 t^{4} D+28 t^{2} D^{3}+768 t^{4}+84 t^{2} D^{2} \\
+88 t^{2} D-D^{3}+32 t^{2}
\end{aligned}
\end{aligned}
$$


Hodge-Tate polynomials. Let $f$ be a Laurent polynomial in 3 variables with Newton polytope $P$, let $F$ be a facet of $P$, and let $f_{F}$ be the corresponding face term. Let $X_{F}$ be the toric variety corresponding to the polygon $F$. The equation $f_{F}=0$ defines a curve in $X_{F}$. If $f$ is a MP then each such curve is of genus zero, thus MPs are Hodge-Tate in the following sense.

Definition 6.5. A 3 -variable Laurent polynomial $f$ with Newton polytope $P$ is Hodge-Tate if for all facets $F \subset P$, the curve $f_{F}=0$ has geometric genus zero.

One might hope that Hodge-Tate polynomials are of low ramification.

Example 6.6. Consider the pictured polygon. This is one of the smallest faces for

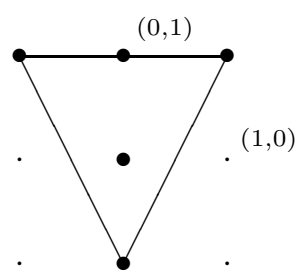

which the Minkowski ansatz has nothing to say. Consider the Laurent polynomial with this Newton polygon given by $f=y\left(x^{-1}+2+x\right)+y^{-1}+a$. For generic $a$ (the completion of) $f=0$ is a curve of geometric genus 1 ; it becomes of genus 0 exactly when $a \in\{-4,0,4\}$. Let us take $a=4$ and use this as a new "puzzle piece" for assembling a Laurent polynomial.

Consider the 3-dimensional reflexive polytope with id 547363 in the GRDB database of toric canonical Fano 3-folds [BK]. This polytope has four faces: two smooth triangles, one $A_{2}$-triangle, and one face isomorphic to the polygon shown above. The corresponding Laurent polynomial is:

$$
F=x+y+z+x^{-4} y^{-2} z^{-1}+2 x^{-2} y^{-1}+4 x^{-1}
$$

It has period sequence:

$$
1,0,8,0,120,0,2240,0,47320,0, \ldots
$$

and Picard-Fuchs operator:

$512 t^{4} D^{3}+3072 t^{4} D^{2}+5632 t^{4} D-48 t^{2} D^{3}+3072 t^{4}-144 t^{2} D^{2}-160 t^{2} D+D^{3}-64 t^{2}$

The Laurent polynomial $F$ is Hodge-Tate but is not a MP. It is extremal, and is of manifold type in the sense of \$7, but is not mirror-dual to any 3-dimensional Fano manifold.

\section{Minkowski polynomials and Fano 3-folds}

Recall that in 3 variables there are 165 Minkowski (classical) period sequences and, correspondingly, 165 Picard-Fuchs operators. We write $L_{f}=\sum t^{k} P_{k}(D)$ where 
$P_{k}(D) \in \mathbb{C}[D]$ is a polynomial in $D$, and denote by $L_{f}(0)=P_{0}(D)$ the operator at $t=0$. It turns out that, if $L_{f}$ is one of the 165 Minkowski Picard-Fuchs operators, then $L_{f}(0)$ splits as a product of linear factors over the rationals. We say that $L_{f}$ is of manifold type if all the roots are integers; otherwise we say that $L_{f}$ is of orbifold type. Exactly 98 of the Minkowski Picard-Fuchs operators are of manifold type and we have verified, by direct computation of invariants on both sides, that they mirror the 98 deformation families of 3-dimensional Fano manifolds $X$ such that $-K_{X}$ is very ample. It will be interesting to see if the Minkowski Picard-Fuchs operators of orbifold type mirror Fano orbifolds.

It is natural to ask what invariants of a Fano manifold $X$ can be computed from the knowledge of the differential operator $\widehat{Q}_{X}$ alone. This is a subtle question EHX97, ES06, but in the case of 3-folds we have good numerical evidence for the following:

Hope 7.1 (Galkin, Golyshev, Iritani, van Straten). Let $X$ be a 3-dimensional Fano manifold and let $J_{X}(t)$ and $J_{X}^{0}(t)$ be as defined above (in the proof of Theorem 4.3). Then:

$$
\lim _{t \rightarrow+\infty} \frac{J_{X}(t)}{J_{X}^{0}(t)}=\widehat{\Gamma}\left(T_{X}\right)
$$

where the limit is taken as $t$ tends to $+\infty$ along the real axis. The characteristic class $\widehat{\Gamma}\left(T_{X}\right)$ is defined in [KKP08, Iri09].

We briefly mention a promising line of thought. Consider a 3-fold toric Gorenstein canonical singularity $X_{\sigma}$, so that $\sigma=\mathbb{R}_{+}(\iota F)$ where $F \subset \mathbb{Z}^{2}$ is a a lattice polygon and $\iota: \mathbb{Z}^{2} \rightarrow \mathbb{Z}^{3}$ is an affine embedding at height one as above. According to Alt97, deformation components of the singularity correspond to Minkowski decompositions of $F$. This suggests that Minkowski polynomials $f$ with Newt $f=P$ may correspond to smoothing components of the singular toric Fano 3 -fold $X$ with fan polytope $P$. It would be nice to make this precise, and to interpret the Minkowski polynomials in terms of holomorphic disk counts in the framework of Hori, Gross-Siebert, or Kontsevich-Soibelman.

\section{Fano 4-folds?}

In 4 dimensions, there are over 473 million reflexive polytopes. Building on the Kreuzer-Skarke classification [KS00, we are now in the process of making a database of facets and of computing their lattice Minkowski decompositions. We plan to classify: Minkowski polynomials (and more general low ramification Laurent polynomials) in 4 variables; their period sequences; and their Picard-Fuchs operators. This will give a list of candidate families of Fano 4-folds, and we aim to: compute the (conjectural) invariants of these Fano 4-folds assuming that they exist; and construct the Fano explicitly in many cases. Eventually, we hope turn this story into a classification theory. 
Acknowledgements. This research is supported by TC's Royal Society University Research Fellowship; ERC Starting Investigator Grant number 240123; the Leverhulme Trust; Kavli Institute for the Physics and Mathematics of the Universe (WPI); World Premier International Research Center Initiative (WPI Initiative), MEXT, Japan; Grant-in-Aid for Scientific Research (10554503) from Japan Society for Promotion of Science and Grant of Leading Scientific Schools (N.Sh. 4713.2010.1); and EPSRC grant EP/I008128/1 "Extremal Laurent Polynomials".

\section{References}

[Alt97] Klaus Altmann. The versal deformation of an isolated toric Gorenstein singularity. Invent. Math., 128(3):443-479, 1997.

[Bat94] Victor V. Batyrev. Dual polyhedra and mirror symmetry for Calabi-Yau hypersurfaces in toric varieties. J. Algebraic Geom., 3(3):493-535, 1994.

[Bea82] Arnaud Beauville. Les familles stables de courbes elliptiques sur $\mathbf{P}^{1}$ admettant quatre fibres singulières. C. R. Acad. Sci. Paris Sér. I Math., 294(19):657660,1982

[B] Borel, A. et al. Algebraic D-modules. Perspectives in Mathematics volume 2, Academic Press Inc., Boston, MA, 1987.

[BH] Lev A. Borisov and R. Paul Horja. On the better behaved version of the GKZ hypergeometric system. arXiv:math.AG/1011.5720.

[BK] Gavin Brown and Alexander M Kasprzyk. The graded ring database homepage. Online. Access via http://grdb.Iboro.ac.uk/

[CG07] Tom Coates and Alexander Givental. Quantum Riemann-Roch, Lefschetz and Serre. Ann. of Math. (2), 165(1):15-53, 2007.

[CG12] John Alexander Cruz Morales and Sergey Galkin. Upper bounds for mutations of potentials. Preprint IPMU 12-0110, 2012.

[Del71] Pierre Deligne. Travaux de Griffiths. In Séminaire Bourbaki. Vol. 1969/70: Exposés 364-381, exp. $n^{\circ}$ 376, volume 180 of Lecture Notes in Mathematics, pages 213-237. Springer-Verlag, 1971.

[Del89] P. Deligne. Le groupe fondamental de la droite projective moins trois points. In Galois groups over $\mathbf{Q}$ (Berkeley, CA, 1987), volume 16 of Math. Sci. Res. Inst. Publ., pages 79-297. Springer, New York, 1989.

[EHX97] Tohru Eguchi, Kentaro Hori and Chuan-Sheng Xiong. Gravitational quantum cohomology. Internat. J. Modern Phys. A, 12(9):1743-1782, 1997.

[ES06] Christian van Enckevort and Duco van Straten. Monodromy calculations of fourth order equations of Calabi-Yau type. In Mirror symmetry. $V$, volume 38 of AMS/IP Stud. Adv. Math., pages 539-559. Amer. Math. Soc., Providence, RI, 2006.

[Giv98] Alexander Givental. A mirror theorem for toric complete intersections. In Topological field theory, primitive forms and related topics (Kyoto, 1996), volume 160 of Progr. Math., pages 141-175. Birkhäuser Boston, Boston, MA, 1998. 
[GKZ08] I. M. Gel'fand, M. M. Kapranov, and A. V. Zelevinsky. Discriminants, resultants and multidimensional determinants. Modern Birkhäuser Classics. Birkhäuser Boston Inc., Boston, MA, 2008. Reprint of the 1994 edition.

[Gol] Vasily Golyshev. Spectra and strains. arXiv:hep-th/0801.0432.

[Gol07] Vasily V. Golyshev. Classification problems and mirror duality. In Surveys in geometry and number theory: reports on contemporary Russian mathematics, volume 338 of London Math. Soc. Lecture Note Ser., pages 88-121. Cambridge Univ. Press, Cambridge, 2007.

[GU10] Sergey Galkin and Alexandr Usnich. Mutations of potentials. Preprint IPMU 10-0100, 2010.

[Gue08] Martin A. Guest. From quantum cohomology to integrable systems, volume 15 of Oxford Graduate Texts in Mathematics. Oxford University Press, Oxford, 2008 .

[GZK89] I. M. Gel'fand, A. V. Zelevinskiǔ, and M. M. Kapranov. Hypergeometric functions and toric varieties. Funktsional. Anal. i Prilozhen., 23(2):12-26, 1989.

[HKTY95] S. Hosono, A. Klemm, S. Theisen, and S.-T. Yau. Mirror symmetry, mirror map and applications to Calabi-Yau hypersurfaces. Comm. Math. Phys., 167(2):301-350, 1995.

[Iri09] Hiroshi Iritani. An integral structure in quantum cohomology and mirror symmetry for toric orbifolds. Adv. Math., 222(3):1016-1079, 2009.

[Isk77] V. A. Iskovskih. Fano threefolds. I. Izv. Akad. Nauk SSSR Ser. Mat., 41(3):516-562, 717, 1977.

[Isk78] V. A. Iskovskih. Fano threefolds. II. Izv. Akad. Nauk SSSR Ser. Mat., 42(3):506-549, 1978.

[Jan90] Uwe Jannsen. Mixed motives and algebraic K-theory, volume 1400 of Lecture Notes in Mathematics. Springer-Verlag, Berlin, 1990. With appendices by S. Bloch and C. Schoen.

[KKP08] L. Katzarkov, M. Kontsevich, and T. Pantev. Hodge theoretic aspects of mirror symmetry. In From Hodge theory to integrability and TQFT tt*-geometry, volume 78 of Proc. Sympos. Pure Math., pages 87-174. Amer. Math. Soc., Providence, RI, 2008.

[KS98] Maximilian Kreuzer and Harald Skarke. Classification of reflexive polyhedra in three dimensions. Adv. Theor. Math. Phys., 2(4):853-871, 1998.

[KS00] Maximilian Kreuzer and Harald Skarke. Complete classification of reflexive polyhedra in four dimensions. Adv. Theor. Math. Phys., 4(6):1209-1230, 2000.

[Man99] Yuri I. Manin. Frobenius manifolds, quantum cohomology, and moduli spaces, volume 47 of American Mathematical Society Colloquium Publications. American Mathematical Society, Providence, RI, 1999.

[MM04] Shigefumi Mori and Shigeru Mukai. Extremal rays and Fano 3-folds. In The Fano Conference, pages 37-50. Univ. Torino, Turin, 2004.

[Prz07] Victor Przyjalkowski. On Landau-Ginzburg models for Fano varieties. Commun. Number Theory Phys., 1(4):713-728, 2007. 
[Sti07] Jan Stienstra. GKZ hypergeometric structures. In Arithmetic and geometry around hypergeometric functions, volume 260 of Progr. Math., pages 313-371. Birkhäuser, Basel, 2007.

[Tak89] Kiyohiko Takeuchi. Some birational maps of Fano 3-folds. Compositio Math., 71(3):265-283, 1989.

Tom Coates, Department of Mathematics, Imperial College London, 180 Queen's Gate, London SW7 2AZ, UK

E-mail: t.coates@imperial.ac.uk

Alessio Corti, Department of Mathematics, Imperial College London, 180 Queen's Gate, London SW7 2AZ, UK

E-mail: a.corti@imperial.ac.uk

Sergey Galkin, Kavli IPMU (WPI), The University of Tokyo, Kashiwa, Chiba 2778583, Japan

E-mail: Sergey.Galkin@ipmu.jp

Vasily Golyshev, Algebra and Number Theory Sector, Institute for Information Transmission Problems, Bolshoy Karetny per. 19, Moscow 127994, Russia

E-mail: platforma.russia@gmail.com

Alexander Kasprzyk, Department of Mathematics, Imperial College London, 180 Queen's Gate, London SW7 2AZ, UK

E-mail: a.m.kasprzyk@imperial.ac.uk 